\title{
Association of Epilepsy and Tourette Syndrome in Children
}

Kun-Long Hung ${ }^{1,2^{*}}$

${ }^{1}$ School of medicine, Fu-Jen Catholic University, New Taipei, Taiwan

${ }^{2}$ Department of Pediatrics, Cathay General Hospital, Taipei, Taiwan

"Corresponding author: Kun-Long Hung, School of medicine, Fu-Jen Catholic University, New Taipei, Taiwan, Tel: 886-2-27082121; Fax: 886-2-27082423; E-mail: klhung0228@gmail.com

Received date: Jun 25, 2017; Accepted date: Jul 03, 2017; Published date: Jul 10, 2017

Copyright: (c) 2017 Hung KL. This is an open-access article distributed under the terms of the Creative Commons Attribution License, which permits unrestricted use, distribution, and reproduction in any medium, provided the original author and source are credited.

\section{Abstract}

Epilepsy is increasingly recognized as an important association with tic disorders and Tourette syndrome in children. Previous studies reported the overall prevalence of Tourette syndrome was around $1 \%$ in children. Epidemiological studies showed evidences of increased neuropsychiatric comorbidities of Tourette syndrome including attention-deficit hyperactivity disorder, obsessive-compulsive disorder and autistic spectrum disorder. The recent studies have demonstrated that increased risk of epilepsy in children with Tourette syndrome, and also increased risk of Tourette syndrome in epilepsy children. The genetic and clinical information refer to a common neurobiological basis for epilepsy and Tourette syndrome.

Keywords: Tic disorder; Tourette syndrome; Epilepsy; Comorbidity; Children

\section{Introduction}

Tourette Syndrome (TS) is a neurodevelopmental disorder characterized by multiple motor tics and one or more vocal tics occurring for at least 1 year, with the onset age before 18 years old. Tic disorders and TS are common in children. Epidemiological studies show that the prevalence of TS is about $1 \%$ in school-age children in western countries, whereas only approximately $0.5 \%$ in Asian countries [1]. The etiology of TS attributes to be multifactorial including genetic, immunological and hormonal factors [2]. Many studies also reported high incidence of neuropsychiatric comorbidities in TS such as Attention-deficit Hyperactivity Disorders (ADHD), Obsessive Compulsive Disorder (OCD) and Autism Spectrum Disorder (ASD) [3]. The presence of comorbidity in TS increases the need for drug and interventional therapy [4]. The comorbidity of TS and epilepsy has been rarely reported.

Rizzo et al. [5] reported a case series of 8 children with TS presenting with the comorbidities of ADHD and epilepsy. Most of these patients developed epilepsy before the onset of tic disorder. They found patients with the triple comorbidity could benefit from anticonvulsants such as topiramate or levetiracetam, as described earlier [6].

The recent 2 nation-wide population-based case-control studies in Taiwan [7,8] investigated the correlation between epilepsy and TS among children by using National Health Insurance Research Database (NHIRD), which has been collecting nation-wide health care data since 1995 when the Taiwan National Insurance (NHI) program was implemented in Taiwan. The results showed a strong correlation between epilepsy and TS. Children with epilepsy had a significantly increased risk of developing TS (adjusted $\mathrm{HR}=8.70,95 \%$ $\mathrm{CI}=4.62-16.37, \mathrm{p}<0.001)$. Meanwhile, there was a significant increase of epilepsy risk in children with Tourette syndrome (adjusted $\mathrm{HR}=16.27,95 \% \mathrm{CI}=6.26-18.46, \mathrm{p}<0.001)$. The studies also showed that children with epilepsy, especially those with intractable epilepsy, have a significantly increased risk of Tourette syndrome. A shared neurobiology of epilepsy and TS was demonstrated. The studies also disclosed that the medication of TS, such as haloperidol, risperidone, sulpiride, clonidine and aripiprazole would not cause the increased risk of epilepsy. Children with TS are likely to develop new-onset epilepsy than general population.

The pathophysiology of epilepsy and TS remains unclear, yet abnormal cortico-basal ganglion circuit connections and dopaminergic systems are possibly involved [9]. Alternations of dopaminergic neurotransmission and corticostriatal network are substantially evident in TS. It is thus reasonable to believe that the correlation exists between epilepsy and TS. As a fact, the potential effectiveness of antiepileptic drugs such as topiramate or levetiracetam in the management of TS indicates that these two disorders may share similar pathophysiologic mechanisms. Recently the modulation of autonomic activity linkage to cognition and behavior was demonstrated in the central thalamocortical regulation, which implicated the additional autonomic role in the regulation of epilepsy and TS [10].

\section{Conclusion}

In conclusion, many case series and population-based studies have explored the correlation between epilepsy and TS. Children with epilepsy have a significantly increased risk of developing TS, whereas, significant increased risk of epilepsy also exists in children with TS. Though there may be a shared neurobiology of epilepsy and TS, further prospective studies to clarify the realistic association between them are mandatory.

\section{References}

1. Robertson MM, Eapen V, Cavanna AE (2009) The international prevalence, epidemiology, and clinical phenomenology of Tourette syndrome: a crosscultural perspective. J Psychosom Res 67: 475-483.

2. Du JC, Chiu TF, Lee KM, Wu HL, Yang YC, et al. (2010) Tourette syndrome in children: an updated review. Pediatr Neonatol 51: 255-264.

3. Cavanna AE, Rickards (2013) The psychopathological spectrum of Gilles de la Tourette syndrome. Neurosci Biobehav Rev 37: 1008-1015.

4. Debes NM, Hjalgrim H, Skov L (2009) The presence of comorbidity in Tourette syndrome increases the need for pharmacological treatment. J Child Neurol 24: 1504-1512.

5. Rizzo R, Gulisano M, Cali PV, Curatolo P (2010) ADHD and epilepsy in children with Tourette syndrome: a triple comorbidity? Acta Paediatr 99: 1894-1896. 
Citation: Kun-Long Hung (2017) Association of Epilepsy and Tourette Syndrome in Children. J Epilepsy 3: 1000e115. doi: 10.4172/2472-0895.1000e115

Page 2 of 2

6. Martinez-Granero MA, Garcia-Perez A, Montanes F (2010) Levetiracetam as an alternative therapy for Tourette syndrome. Neuropsych Dis Treat 6 309-316.

7. Weng WC, Huang HL, Wong LC, Jong YJ, Yin YJ, et al. (2015) Increased risks of tic disorders in children with epilepsy: a nation-wide populationbased case-control study in Taiwan. Res Dev Disabil 51-52: 173-180.
8. Wong LC, Huang HL, Weng WC, Jong YJ, Yin YJ, et al. (2015) Increased risk of epilepsy in children with Tourette syndrome: a population-based case-control study. Res Dev Disabil 51-52: 181-187.

9. Starr MS (1996) The role of dopamine in epilepsy. Synapse 22: 159-194.

10. Nagai Y (2015) Modulation of autonomic activity in neurological conditions: epilepsy and Tourette syndrome. Front Neurosci 9: 278. 